\title{
The Brown Dwarf Deficit in Taurus: Evidence for a Non-Universal IMF
}

\author{
César Briceño \\ Centro de Investigaciones de Astronomía (CIDA), Apartado Postal 264, \\ Mérida 5101-A, Venezuela \\ Kevin L. Luhman \& Lee Hartmann \\ Harvard-Smithsonian Center for Astrophysics, 60 Garden St., \\ Cambridge, MA 02138, USA \\ John R. Stauffer \\ SIRTF Science Center, MS 220-6, Caltech, Pasadena, CA 91125, USA \\ J. Davy Kirkpatrick \\ IPAC, MS 100-22, 770 S. Wilson, Caltech, Pasadena, CA 91125, USA
}

\begin{abstract}
We present the results of a deep, optical/IR wide field imaging survey of selected fields in the nearby (d 140 pc) Taurus star-forming region. We report the discovery of 9 new members with spectral types M5.75-M9.5. We derive an Initial Mass Function encompassing $54 \%$ of the known members in Taurus. Comparison with dense regions like the Trapezium Cluster in Orion shows that Taurus has produced $\times 2$ less brown dwarfs. We suggest that the lower frequency of brown dwarfs in Taurus may result from the low-density star-forming environment, leading to larger minimum Jeans masses.
\end{abstract}

\section{Introduction}

The variation of the Initial Mass Function (IMF) with the environment is a key problem in star formation, but any dependence of the IMF with the ambient conditions has been difficult to determine. Most surveys have concentrated on the most compact regions with the highest density of stars and gas, while extended, sparse groups of nearby young stars have not been studied with the same sensitivity, mainly because of the large area of the sky that they span. Therefore, meaningful comparisons of the IMF in differing conditions have not been readily available. This situation is changing with the advent of largeformat CCD detectors, making possible deep surveys of the less embedded, more extended star-forming complexes. With a modest extinction $(\mathrm{Av} \lesssim 4)$ and low density of stars $\left(\mathrm{N} \sim 1-10 \mathrm{pc}^{-3}\right)$ Taurus is an example of the "non-clustered" mode of star formation, making it an ideal laboratory for comparison with high density regions like young clusters. 

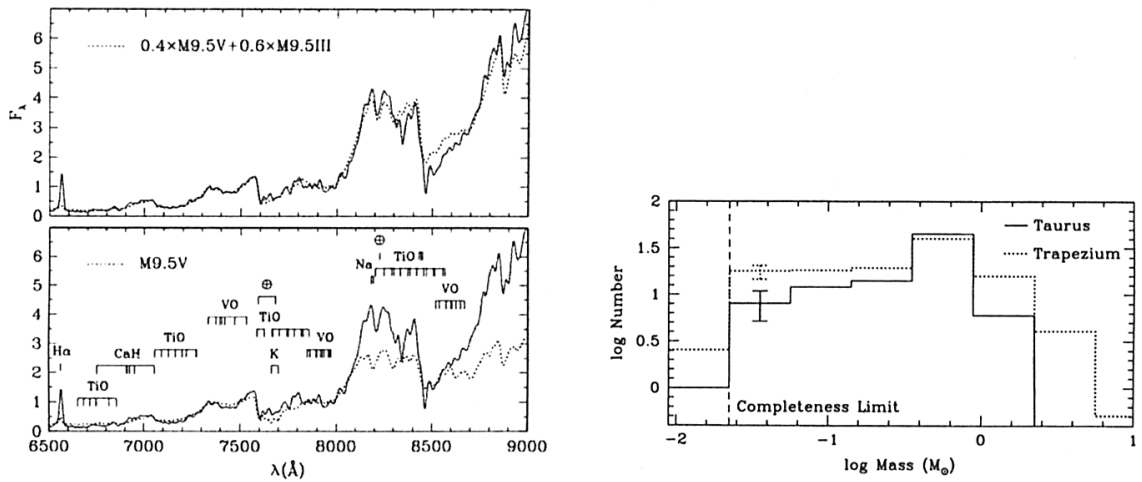

Figure 1. (a) Spectra for the M9.5 brown dwarf. The best fit spectrum (upper panel) is obtained for an intermediate gravity between dwarfs and giants. (b) IMFs for reddening limited samples in Taurus (solid line) and the Trapezium Cluster (dotted line). The Taurus sample was normalized to the Trapezium.

\section{Observations and Results}

We used the $8 \mathrm{k} \times 8 \mathrm{k}$ CCD Mosaic Camera with $I, z^{\prime}$ filters on the KPNO $0.9 \mathrm{~m}$ telescope $\left(1^{o} \times 1^{o} \mathrm{FOV}\right)$ to observe eight fields in Taurus to an $I_{\text {lim }} \sim 21$. With $I, z^{\prime}$ photometry and $J H K_{s}$ data from the 2MASS, we performed a reddening limited $(\mathrm{Av} \lesssim 4)$ search for candidate young ( $\$ 10 \mathrm{Myr})$ low-mass stars and brown dwarfs down to $0.02 \mathrm{M}_{\odot}$ (details in Briceño et al. 2002). We then used the $\mathrm{K}$ and $\mathrm{Na}$ gravity-sensitive features in follow up spectra to confirm membership.

Among the new members is an M9.5 brown dwarf (Figure 1a), the least massive object identified so far in Taurus $\left(15 \mathrm{M}_{\text {Jup }}\right.$ using the temperature scale of Luhman [2000] and evolutionary tracks of Baraffe et al. [1998]). This object exhibits significant near-IR excess and strong $\mathrm{H} \alpha$ emission $(\mathrm{W}[\mathrm{H} \alpha]=150 \AA)$, indicative of ongoing accretion from a circumstellar disk and evidence that brown dwarfs, like stars, can form from fragmentation processes.

We construct an IMF for our Taurus fields and compare it with the IMF for the Trapezium Cluster (Figure 1b). We find that the ratio of substellar to stellar objects in the Trapezium is $\times 2$ larger than in Taurus; the scarcity of brown dwarfs may be due to the higher value of the Jeans mass in Taurus, which results from the lower surface density of gas in this region.

\section{References}

Baraffe, I., Chabrier, G., Allard, F., \& Hauschildt, P. H., 1998, A\&A, 337, 403

Briceño, C., Luhman K., Hartmann, L., Stauffer, J., Kirkpatrick, D., 2002, ApJ, in press.

Luhman, K., 2000, ApJ, 544, 104 